\title{
Incomplete contacts in partial slip subject to varying normal and shear loading, and their representation by asymptotes
}

\author{
R.M.N. Fleury ${ }^{\mathrm{a}, *}$, D.A. Hills ${ }^{\mathrm{a}}$, R. Ramesh ${ }^{\mathrm{a}}$, J. R. Barber ${ }^{\mathrm{b}}$ \\ ${ }^{a}$ Department of Engineering Science, University of Oxford, Parks Road, OX1 3PJ, Oxford, UK. \\ ${ }^{b}$ Department of Mechanical Engineering, University of Michigan, Ann Arbor, MI 48109-2125, USA.
}

\begin{abstract}
We develop a method for the solution of partial slip contact problems suffering complex loading cycles where, generally, the normal load, shear force and, potentially, differential bulk tensions are all functions of time, using an edge-asymptote approach. The size of the slip zone and local shear traction distribution are revealed as functions of time. The results are then re-worked in asymptotic form, so that they do not hinge on inherent symmetry and antisymmetry conditions for the contact overall, and are of general applicability. The multipliers on the local solutions (generalised stress intensity factors) are also appropriate as a means of taking laboratory tests quantifying fretting fatigue and employing them to wholly different prototypical problems.
\end{abstract}

\section{Introduction}

Fretting fatigue, the accelerated nucleation of cracks caused by small amounts of differential movement between components pressed together, is conveniently divided into two phases; the nucleation of cracks and their propagation. Here, a rigorous quantification of the edge slip conditions for incomplete (convex) contacts is sought. The results to be derived are applicable to any geometry of contact which is capable of local idealization using half-plane analysis, i.e. to almost any contact which advances as the normal load is increased; it is necessary neither to have a closed form solution for the contact, nor even for the contact as a whole to be capable of idealization by half plane (or space) theory. The results found can be used in conjunction with geometries where only a numerical method such at the finite element procedure may be used to solve the contact problem, and which may be used to abstract the generalized stress intensity factors required as inputs here. In this paper, however, they are applied to simple problems where closed form algebra is possible for comparison.

The first solution for partial slip in contact was by Cattaneo who studied the Hertz problem [1]. Mindlin independently found the same solution many years later [2] and went on to look at further loading paths [3,4]. Around the same time, Galin $[5,6]$ solved the partial slip problem of a rigid flat punch indenting an elastic half-plane. The next development was the inclusion of the effects of differential tension [7], but a big development came when, independently, Jäger [8] and Ciavarella [9] noted that, for any geometry of half-plane contact, the corrective shear traction in the stick region was a scaled form of the sliding distribution. This has proved useful in many partial slip solutions, and a simplified notation developed by Barber et al. [10] enabled the problem of partial slip problems involving varying normal load to be attacked. Separately, partial slip edge solutions were developed from the Cattaneo solution but where the edge direct and shear tractions were captured using asymptotic forms [11, 12]. These followed the work of Sackfield et al. [13] who first introduced asymptotic solutions to characterising the edge of contacts. This has the big advantages that they are (a) independent of the overall contact geometry, (b) remove the need for symmetry/antisymmetry across the whole contact, and (c) as a consequence of (a) may be used as quantifiers of fretting fatigue enabling laboratory specimens test to be applied to very different problems [14, 15]. The Ciavarella-Jäger theorem

\footnotetext{
${ }^{*}$ Corresponding author. Fax: +44 01865273906

Email addresses: rodolfo.fleury@eng.ox.ac.uk (R.M.N. Fleury), david.hills@eng.ox.ac.uk (D.A. Hills), rangarajan.ramesh@eng.ac.uk (R. Ramesh), jbarber@umich.edu (J. R. Barber)
} 
cannot be used when the slip zones at each end of the contact have opposite signs. This restriction is removed if we concentrate our attention on one end only. One possibility explored here is to represent the behaviour of each end by a set of simple asymptotic forms.

The step made in [10] was very significant and helpful in solving a number of real partial slip problems where the normal load varied, but these solutions suffered from the inherent limitation that the shear traction had to have its origin in the form of an applied shear force only whereas, in practice, differential bulk tensions in the bodies is equally important. The equivalence of the two methods of excitation for a constant normal load was discussed exhaustively in [16] for the case of a constant normal load, and here, we combine the concepts in [10] and [16] to enable us to solve for edge slip in contacts where normal load, shear force and (potentially) bulk tension all vary in time. The method hinges on taking the results in [10], attaching asymptotic forms to them, and noting that the origin of the shear stress intensity factor may be from either a shear force or differential tension.

Initially, we assume that an incomplete contact of half-width $a$ is fully stuck, so that the local shearing traction distribution, $q(x)$, at the left hand edge of the contact, $x \rightarrow-a^{+}$, is given by

$$
q(x)=\frac{K_{T}}{\sqrt{x+a}}=\frac{K_{T}}{\sqrt{s}},
$$

where $s=a+x$ (see Fig. 1) and the generalised stress intensity factor, $K_{T}$, may be induced by a shear force, $Q$, or a differential bulk tension, $\sigma_{0}$. The generalised stress intensity factor $K_{T}$ can be excited by the shear traction and/or a differential bulk tension and is given by ${ }^{1}$

$$
K_{T}=\frac{ \pm Q}{\pi \sqrt{2 a}}+\frac{\sigma_{0}}{2} \sqrt{\frac{a}{2}} .
$$

Note that this result is wholly independent of the geometry of the problem in cases where the body may be thought of as a half-plane. Where the contact geometry is such that only local half-plane behaviour arises, such as a pin located in an almost conforming hole, more complicated definitions will be needed. The contact problem will be inherently uncoupled, and so we may write the local contact pressure at the end $x \rightarrow-a^{+}$in the form

$$
p(x)=K_{N} \sqrt{a+x}=K_{N} \sqrt{s} .
$$

Here the value of the generalized stress 'intensity' factor is geometry dependent. Interestingly, the same information is incorporated into the instantaneous contact law, $a(P)$, and it may be shown (see Appendix $\mathrm{A}$ ) that

$$
K_{N}=\frac{1}{\pi} \sqrt{\frac{2}{a}} \frac{\mathrm{d} P}{\mathrm{~d} a},
$$

where $P$ is the normal load on the contact giving rise to a contact of half-width $a$. Note that only a limited number of problems will have a closed form contact law. For these an analytical equation for $K_{N}$ may also be obtained. Example calibrations of $K_{N}$ were summarised in [16]. For more complex problems a semi-analytical formulation may be obtained by using the finite element method to find the calibration. We also record that the calibration of the problem of an elastic flat and rounded semi-infinite punch was obtained in $[17,18]$. The other pre-requisite for this paper is an understanding of the Ciavarella-Jäger theorem applied to a problem defined in terms of these asymptotic forms, and this is set out in Appendix B.

These edge asymptotes are illustrated in Fig. 1. Note, however, that there is one more possible form of asymptote needed, and which arises when both the shear and normal forces applied to the contact change, but $|\mathrm{d} Q / \mathrm{d} P|<f$. In this case the additional near-edge shear traction is given by

$$
q(x)=K_{Q} \sqrt{s}
$$

\footnotetext{
${ }^{1}$ See [16] for a discussion of the sign conventions employed at each edge of the contact.
} 


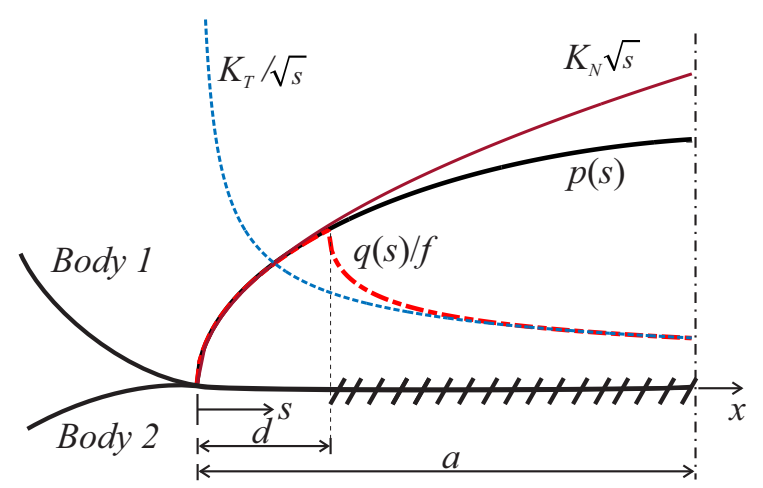

Figure 1: Normal square root bounded asymptote and tangential square root singular asymptote.

where the generalised intensity factor $K_{Q}$ may be obtained from the normal generalised stress intensity factor as

$$
K_{Q}=\left( \pm \frac{\mathrm{d} Q}{\mathrm{~d} P}+\frac{\pi a}{4} \frac{\mathrm{d} \sigma_{0}}{\mathrm{~d} P}\right) K_{N}
$$

A more general representation of the bounded shear intensity factor, $K_{Q}$, was also given in [19] but, although more restrictive in its range of application, Eq. (6) is more convenient to use and provides a good approximation in the range where the asymptotic approach is valid. Although the effect of the bulk tension may be easily included in the asymptotic approach through Eqs. (2) and (6), in the remainder of this paper we will be looking only at the effect of a shear force. This is partly because we are interested in the accuracy of the asymptotic solution compared with the exact solution and the latter is only available in the absence of tension. Although the quality of the asymptotes is problem specific, it is shown in Section 5 that the asymptotic approach gives good approximate results (within 10\%) of the slip zone size for Hertzian contacts if the load ratio do not exceed $Q / P=0.33$.

\section{Varying normal and shear load problem - finite contact solution}

The problem of alternating periodic shear and normal loads, applied initially to a general incomplete contact, is investigated here. This can be thought of as an 'inclined' Cattaneo-Mindlin problem (in $P-Q$ space), where the normal load also varies periodically and in phase with the shear traction. An initial loading from $\mathrm{O}$ to A is applied first and only then does the periodic loading begin (Fig. 2). This initial loading can take any trajectory, as long as the full stick condition is satisfied, i.e. $|d Q / d P|<f$. Note that, when the initial shear loading is zero, and the normal load is subsequently held constant, this becomes the standard Cattaneo-Mindlin problem. The periodic loading $P(t)$ and $Q(t)$ are, generally, of the form

$$
\begin{aligned}
& P(t)=P_{0}+P_{a} \sin (\omega t+\phi) \\
& Q(t)=Q_{0}+Q_{a} \sin \omega t
\end{aligned}
$$

but here the normal and shear loads are in phase $(\phi=0)$ and the mean values of the periodic cycle are those at the end of the initial loading phase, i.e. $P_{0} \equiv P_{A}$ and $Q_{0} \equiv Q_{A}$ and, of course, $P_{a} \equiv \Delta P / 2$ and $Q_{a} \equiv \Delta Q / 2$. The shear traction distributions for periodic loading with varying normal and shear tractions have been described in detail by Barber et al. [10]. The shear traction for the finite problem is presented first for clarity, and for the purpose of assessing the quality of the asymptotic representation in Section 3, when specialised to a Hertzian contact.

\subsection{First cycle - loading from A to B}

When the contact is loaded from A to B $\left(Q=Q_{\max }\right)$ it forms the monotonically increasing part of the transient load cycle, and the shear traction distribution found is similar to the standard Cattaneo-Mindlin problem at a constant normal load $P_{\text {max }}$. Throughout this paper it is assumed that the friction law is given by the Amontons-Coulomb law 


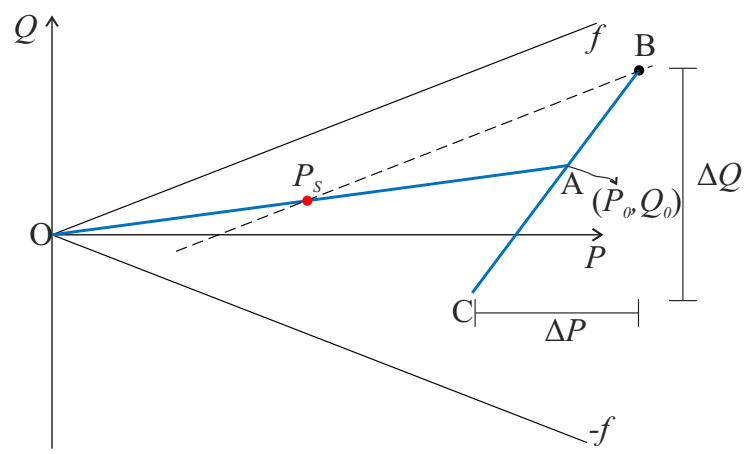

Figure 2: Load path and magnitude of the corrective term, $P_{S}$, during the transient cycle from A to B.

and the limiting friction force when sliding occurs is given by the normal force scaled by a coefficient of friction, $f$ (i.e. $Q=f P$ ). The full solution of the shear traction at a general point $X$ between $\mathrm{A}$ and $\mathrm{B}$ is given by

$$
q_{X}(x, P)=\int_{0}^{P_{S}} \frac{\partial p(x, P)}{\partial P} \frac{\mathrm{d} Q}{\mathrm{~d} P} \mathrm{~d} P+f p\left(x, P_{X}\right)-f p\left(x, P_{S}\right)
$$

where $P_{S}$ is found by imposing tangential equilibrium, which gives $Q_{X}=Q_{S}-f P_{S}+f P_{X}$. This is equivalent to defining the intersection with the initial loading path of a line of slope $f$ passing through a point $\mathrm{X}$ lying between $\mathrm{A}$ and $\mathrm{B}$. The graphical representation of tangential equilibrium when $\mathrm{X}$ has reached point $\mathrm{B}$ is presented in Fig. 2. The second and third terms of Eq. (9) are applied over the total area of the contact, $a_{X}=a\left(P_{X}\right)$, and over the stick zone, $a_{S}=a\left(P_{S}\right)$, respectively. The integral term of Eq. (9) corresponds to the locked in shear traction established inside the stick zone, $a_{S}$, due to the initial loading phase OA, although the part of the locked-in shear defined between $P_{S}$ and $P_{A}$ is erased by slip. The presence of a locked-in shear traction during OA will change the slip zone size during the transient loading cycle but, it will turn out, will have no influence in the steady-state loading cycles.

\subsection{First cycle - unloading from $B$ to $C$}

When the load reaches its maximum value and an infinitesimal decrement is applied, the contact becomes instantaneously fully stuck. Then, as the load is decreased by a finite amount, a reverse slip zone develops from the edge of the contact inwards, similarly to what was shown for a constant normal load problem in [20]. The solution of the shear traction distribution during the unloading phase is given by superposition of a second partial slip problem on Eq. (9), which can also be constructed using the Ciavarella-Jäger theorem and written as

$$
q_{c o r r}=-\left[f p\left(x, P_{X}\right)-f p\left(x, P_{S}\right)\right]+\left[f p\left(x, P_{S}\right)-f p\left(x, P_{Z}\right)\right],
$$

where the first partial slip problem has the terms of the current loading, $P_{X}$, and the new stick zone size $a_{S}$. The second, takes into account the locked in shear in Eq. (9) and is given by a partial slip problem of contact size $a_{S}$ and stick zone size $a_{Z}=a\left(P_{Z}\right)$, where $a_{S}$ defines the stick zone during the unloading phase, and $a_{Z}$ defines the locked-in shear traction in the stick zone at point B. Note that we use the convention that the subscript $S$ always defines the current stick zone and, in this phase of the loading, $P_{Z}$ is the point formerly designated by $P_{S}$ at point $\mathrm{B}\left(P_{Z}=P_{S}^{(B)}\right)$. The shear traction distribution during the unloading phase is then written as

$$
q_{X}(x, P)=\int_{0}^{P_{Z}} \frac{\partial p(x, P)}{\partial P} \frac{\mathrm{d} Q}{\mathrm{~d} P} \mathrm{~d} P-f p\left(x, P_{X}\right)+2 f p\left(x, P_{S}\right)-f p\left(x, P_{Z}\right) .
$$

The moving stick zone during the unloading phase is given by $a_{S}$, where $P_{S}$ is again obtained by imposing tangential equilibrium, i.e. $Q_{X}=Q_{Z}-f P_{X}+2 f P_{S}-f P_{Z}$. This is equivalent to locating the intersection of a line of slope $-f$ passing through the point $\left(P_{X}, Q_{X}\right)$ and a line between point $P_{B}$ and $P_{Z}$ of slope $f$ (Fig. 3a).

Equation (11) is true during the unloading phase as long as $P_{S} \geq P_{Z}$. As the loads continue to decrease, the size of the stick zone, $a_{S}$, also reduces. If the slip zone now extends beyond the previous stick zone at point $\mathrm{B}$, the 


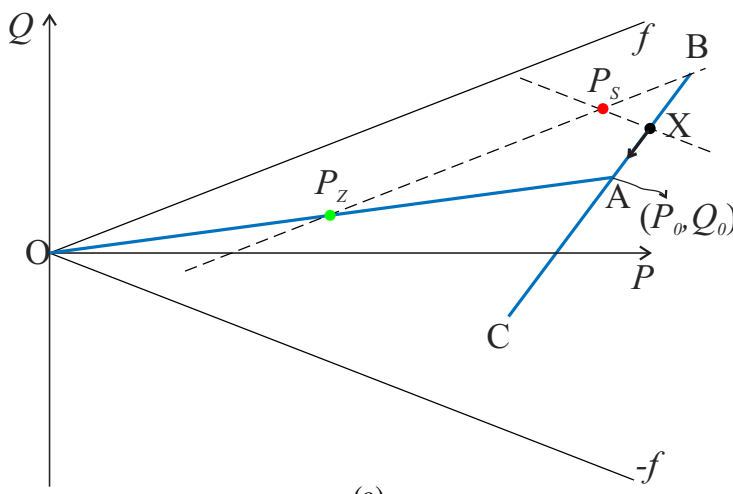

(a)

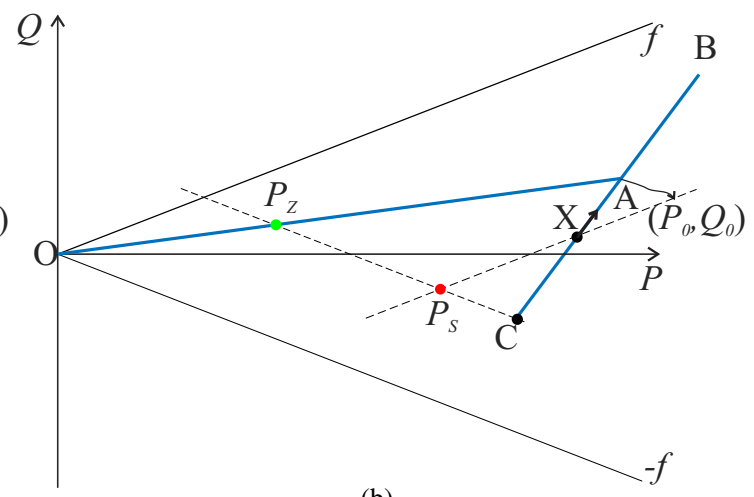

(b)

Figure 3: (a) Load path during the transient cycle from B to C (and $\left.P_{S} \leq P_{Z}\right)$; and (b) the steady-state cycle at $\mathrm{B}^{(S)}\left(Q=Q_{\max }\right.$ and $\left.P=P_{\max }\right)$.

information locked-in the original stick zone is erased and the problem now is identical to the case where the loading is taken directly from point $\mathrm{A}$ to point $\mathrm{C}$. This is also equivalent to a Cattaneo-Mindlin problem of constant normal load $P=P_{\min }$. The shear tractions at this point are given by Eq. (9), but with a change of signs in the second and third terms. The minimum value of $P_{S}$ (which will also define $P_{Z}$ from this point forward) is obtained from tangential equilibrium, $Q_{X}=Q_{Z}-f P_{X}+f P_{Z}$.

\subsection{Steady-state - loading from $C$ to $B$}

When re-loading from $\mathrm{C}$ to B (i.e from $Q_{\min }$ to $Q_{\max }$ ) the contact will also be instantaneously stuck at first, and then forward slip zones will develop at the edges and they will increase in size as the load is increased from $Q_{\text {min }}$ to $Q_{\max }$. This is analogous to the process described in Section 2.2. The shear tractions during this loading phase are given by Eq. (11) and when the normal and shear loads reach B $\left(P_{\max }, Q_{\max }\right)$ again, the shear traction is given by

$$
q_{B}^{(s)}(x, P)=\int_{0}^{P_{Z}} \frac{\partial p(x, P)}{\partial P} \frac{\mathrm{d} Q}{\mathrm{~d} P} \mathrm{~d} P+f p\left(x, P_{B}\right)-2 f p\left(x, P_{S}\right)+f p\left(x, P_{Z}\right) .
$$

The size of the stick zone may again be obtained by imposing tangential equilibrium, as in Section 2.2, and a graphical representation of the different terms is shown in Fig. 3b. Note that, whilst loading from $\mathrm{C}^{(T)}$ to $\mathrm{B}^{(S) 2}$ the stick zone size, $a_{S}$, reduces in size, but now remains always greater than the size of the stick zone at $\mathrm{C}^{(T)}\left(\right.$ i.e. $\left.P_{S}>P_{Z}\right)$. Therefore, there is a zone within the contact, $a\left(P_{S}\right) \backslash a\left(P_{Z}\right)$, that experienced slip in the reverse direction at $\mathrm{C}^{(T)}$, but will not slip in the forward direction during this loading phase. At this point, the problem has reached steady-state and the shear tractions and slip stick behaviour at B will be identical in subsequent cycles.

\subsection{Steady-state - unloading from $B$ to $C$}

During the unloading phase in the steady-state, a reverse slip zone increases from the edge inwards as the load goes to $\left(P_{\min }, Q_{\min }\right)$. Because, at point $\mathrm{B}^{(S)}$, the $P_{S}$ term never cancels out the $P_{Z}$ term, those two terms will be carried forward to the unloading phase. However, the stick zone size will no longer be controlled by the third term of Eq. (12) and we define $a_{K}=a\left(P_{K}\right)$ as the stick zone size at point $\mathrm{B}^{(S)}$. Superposition of another partial slip problem using arguments similar to ones given in Section 2.2, shows that the shear traction during the unloading phase is given by

$$
q_{X}^{(s)}(x, P)=\int_{0}^{P_{Z}} \frac{\partial p(x, P)}{\partial P} \frac{\mathrm{d} Q}{\mathrm{~d} P} \mathrm{~d} P-f p\left(x, P_{X}\right)+2 f p\left(x, P_{S}\right)-2 f p\left(x, P_{K}\right)+f p\left(x, P_{Z}\right) .
$$

The stick zone size, $a_{S}$, can be obtained by defining $P_{S}$ through tangential equilibrium (Fig. 4). As the load approaches point C $\left(P_{\min }, Q_{\min }\right)$, the stick zone size, $a_{S}$, approaches $a_{K}$. At point $\mathrm{C}^{(S)}$, it is clear from Eq. (13) that the $P_{S}$ and $P_{K}$

\footnotetext{
${ }^{2}$ Henceforth, the superscript $(T)$ stands for 'transient' cycle and $(S)$ for 'steady-state' cycle.
} 
terms cancel out and the shear traction is given by

$$
q_{C}(x, P)=\int_{0}^{P_{Z}} \frac{\partial p(x, P)}{\partial P} \frac{\mathrm{d} Q}{\mathrm{~d} P} \mathrm{~d} P-f p\left(x, P_{C}\right)+f p\left(x, P_{Z}\right)
$$

At point $\mathrm{C}^{(S)}$, the stick zone size tends to $a_{K}$, where $P_{S}=P_{K}$. This is evident when observing the extent of the stick zone at a point infinitesimally before $\mathrm{C}^{(S)}$ (Fig. 4). Although the shear traction reaches the sliding limit (i.e. $q=f p$ ) throughout $a(P) \backslash a\left(P_{Z}\right)$ in Eq. (14), the zone $a\left(P_{K}\right) \backslash a\left(P_{Z}\right)$ does not experience further slip during the steady-state phase (this will be later illustrated in Fig. 7). The amount of reverse slip that occurred at $\mathrm{C}^{(T)}$ is still trapped in that zone, since it did not subsequently slip forward at point $\mathrm{B}^{(S)}\left(P_{\max }, Q_{\max }\right)$.

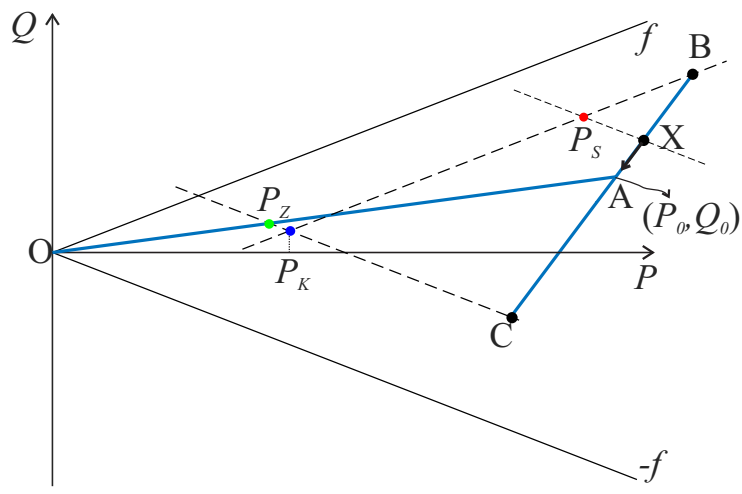

Figure 4: Load path during the steady-state cycle from $\mathrm{B}^{(S)}$ to $\mathrm{C}^{(S)}\left(Q=Q_{\max }\right.$ to $\left.Q=Q_{\min }\right)$.

\section{Varying normal load problem - asymptotic formulation}

The solution presented above, which is a re-write of the solution in [10] for in phase loading $(\phi=0)$, requires a knowledge of the contact law and pressure distribution of the contact. These are often not known in closed form for several types of contacts, e.g. the elastic flat-and-rounded punch on an elastic plane or problems where halfplane approximation is not valid at all points within the contact. In re-writing the contact problem in terms of edge asymptotes, the formulation can be made more easily applicable to complex geometries as long as the contact is incomplete. The generalised stress intensity factors and their corresponding normal and shear tractions are displayed in Fig. 1. Note that although the shear traction singular multiplier $K_{T}$ is geometry independent, the normal multiplier $K_{N}$, and therefore also $K_{Q}$, will depend on the geometry of the punch.

One of the main differences between the asymptotic solution and the solution presented in Section 2, is that tangential equilibrium cannot be used to determine the size of the stick zone. That is because the asymptotic approach does not 'see' the total loading in the contact. Instead, the approach introduced by Dini et al. [11] of matching the inner and outer asymptotes is used, although now we must allow for the change in contact size during the periodic loading as well. Therefore, the focus here is on obtaining an approximate value of the slip zone during the different phases of loading in order to be able to quantify: (i) the quality of the asymptotic approximation; and (ii) the damage one might expect in the different contact zones with different slip/stick behaviour throughout the cyclic loading.

\subsection{First cycle - loading from A to B}

From point A to point $\mathrm{B}$ the contact increases in size. The change in shear traction in the slip zone during this step is given in terms of edge asymptotes as

$$
\Delta q_{A B}=f K_{N}^{B} \sqrt{s_{B}}-K_{Q}^{A} \sqrt{s_{A}}, \quad 0 \leq s_{B} \leq d_{B}
$$

where $K_{N}^{B}=K_{N}\left(P_{B}\right)$ is the normal edge intensity factor due to $P_{B}$ and $K_{Q}^{A}=K_{Q}\left(P_{A}\right)$ is the bounded shear intensity factor at $P_{A}$ due to the initial loading OA. The origins of the sets of coordinates are set at the left hand edges of the 
contacts, and the changes of coordinates are $s_{A}=x+a_{A}$ and $s_{B}=x+a_{B}$ for points $\mathrm{A}$ and $\mathrm{B}$, respectively. Note that the coordinate $s$ is now not fixed in space, but moves with the change in contact size. Equation (15) is re-written in terms of the coordinate $s_{B}$, by making the substitution $s_{B}=s_{A}+\Delta a$, where $\Delta a>0$ for advancing contacts and $\Delta a<0$ for receding contacts. Equation (15) therefore becomes

$$
\begin{aligned}
\Delta q_{A B} & =f K_{N}^{B} \sqrt{s_{B}}-K_{Q}^{A} \sqrt{s_{B}-\Delta a} \\
& \simeq\left(f K_{N}^{B}-K_{Q}^{A}\right) \sqrt{s_{B}}+K_{Q}^{A} \frac{\Delta a}{2 \sqrt{s_{B}}},
\end{aligned}
$$

where the term $\sqrt{s_{B}+\Delta a}$ was expanded using a binomial series and the higher order terms neglected. This is a good approximation only when the change in contact size is much smaller than the mean size of the contact, $\Delta a \ll a\left(P_{0}\right)$. Using the Ciavarella-Jäger theorem on the square root bounded term by superimposing a scaled sliding solution with the origin offset by $d_{B}$, where $d_{B}$ is the slip zone size at $\mathrm{B}$, we obtain

$$
\begin{aligned}
\Delta q_{A B} & =\left(f K_{N}^{B}-K_{Q}^{A}\right)\left(\sqrt{s_{B}}-\sqrt{s_{B}-d_{B}}\right)+K_{Q}^{A} \frac{\Delta a}{2 \sqrt{s_{B}}} \\
& \simeq\left(f K_{N}^{B}-K_{Q}^{A}\right)\left(\frac{d_{B}}{2 \sqrt{s_{B}}}+\ldots\right)+K_{Q}^{A} \frac{\Delta a}{2 \sqrt{s_{B}}} .
\end{aligned}
$$

Note that the change in $K_{N}$ between the contact size, $a_{B}$, and the stick zone size, $a_{B}-d_{B}$, was neglected, since it is assumed the slip zone size is small when compared to the size of the contact for the asymptotes to be valid. By making use of Eq. (1) when $s_{B} \gg d_{B}$ and matching the inner and outer asymptotes, the slip zone size, $d_{B}$, is given by ${ }^{3}$

$$
d_{B}=\frac{2 K_{T}(\Delta Q)-K_{Q}^{A} \Delta a}{f K_{N}^{B}-K_{Q}^{A}} .
$$

The shear traction is then given by

$$
q_{B}(x)=K_{Q}\left(P_{Z}\right) \sqrt{s_{B}-d_{B}}+f K_{N}^{B} \sqrt{s_{B}}-f K_{N}^{B} \sqrt{s_{B}-d_{B}} .
$$

Note that, to simplify the appearance, the term $P_{Z}$ is written in Eq. (19), although $P_{Z}$, here, is not the same as the one in Section 2, since it is not obtained from tangential equilibrium. Instead, $P_{Z}$ here is the 'fictitious' load that will result in a contact size $a_{B}-d_{B}$. Since $K_{Q}$ may be obtained directly from the contact size, there is no need, other than for simplification of the notation, to obtain the equivalent $P_{Z}$ for the solution of the locked in shear traction inside the stick zone $s_{B}>d_{B}$.

\subsection{First cycle - unloading from $B$ to $C\left(Q_{\max }\right.$ to $\left.Q_{\min }\right)$}

The shear traction distribution, in terms of asymptotes, between points $\mathrm{B}^{(T)}$ and $\mathrm{C}^{(T)}$, where $d Q / d t<0$, may be obtained directly from Eq. (11) (although a similar argument to the one made Section 3.1 using the Ciavarella-Jäger theorem could be used as well) as

$$
q_{C}=K_{Q}\left(P_{Z}\right) \sqrt{s_{C}-d_{C}}-f\left(K_{N}^{C} \sqrt{s_{C}}-2 K_{N}^{C} \sqrt{s_{C}-d_{C}}+K_{N}^{C} \sqrt{s_{B}-d_{B}}\right) .
$$

The change in shear traction at a point $X$ when unloading from $\mathrm{B}^{(T)}$ to $\mathrm{C}^{(T)}$ is given by

$$
\Delta q_{B X}=-f K_{N}^{X}\left(\sqrt{s_{X}}-2 \sqrt{s_{X}-d_{X}}+\sqrt{s_{B}-d_{B}}\right)-f K_{N}^{B}\left(\sqrt{s_{B}}-\sqrt{s_{B}-d_{B}}\right) .
$$

\footnotetext{
${ }^{3}$ Here, the singular scaling factor $K_{T}(\Delta Q)$ is given by Eq. (2) but with the variation $\Delta Q$ instead of $Q$. This is not the same as $\Delta K_{T}$, which is the variation of $K_{T}$ between two different load instants.
} 
If the change in normal generalised stress intensity factor is neglected, $K_{N}^{X} \simeq K_{N}^{B}$, then we may rewrite Eq. (21) as

$$
\begin{aligned}
\frac{\Delta q_{B X}}{f K_{N}^{X}} & =-\sqrt{s_{X}}+2 \sqrt{s_{X}-d_{X}}-\sqrt{s_{X}-\Delta a} \\
& \simeq-2\left(\frac{d}{2 \sqrt{s_{X}}}+\ldots\right)+\frac{\Delta a}{2 \sqrt{s_{X}}} .
\end{aligned}
$$

By making use of Eq. (1) when $s \gg d$, we may write the size of the reverse slip zone as

$$
d_{X}=-\frac{\Delta K_{T}}{f K_{N}^{X}}+\frac{\Delta a}{2} .
$$

Note that during the unloading phases $\Delta K_{T}<0$ and the negative sign makes $d_{X}$ positive in Eq. (23).

When the size of $a_{X}-d_{X}$ reaches $a_{B}-d_{B}$ (equivalent to when $P_{S} \leq P_{Z}$ in the full solution formulation), the locked-in slip at point $\mathrm{B}$ is erased and the problem becomes equivalent to a Cattaneo-Mindlin problem of load $P_{C}$. Hence, the slip zone size is obtained in a similar manner to that from point A to point $\mathrm{B}$ as

$$
d_{C}=-\frac{2 K_{T}(\Delta Q)-K_{Q}^{A} \Delta a}{f K_{N}^{C}+K_{Q}^{A}},
$$

and the shear traction is given by

$$
q_{C}=f K_{N}^{C} \sqrt{s_{C}}-f K_{N}^{C} \sqrt{s_{C}-d_{C}} .
$$

\subsection{Steady-state - loading from $C$ to $B\left(Q_{\min }\right.$ to $\left.Q_{\max }\right)$}

The shear traction distribution at a point $X$ between the points $\mathrm{C}^{(T)}$ and $\mathrm{B}^{(S)}$ (Fig. 3b) can be obtained by rewriting Eq. (12) in asymptotic form as

$$
q_{X}(x)=K_{Q}\left(P_{Z}\right) \sqrt{s_{C}-d_{C}}+f K_{N}^{X}\left(\sqrt{s_{X}}-2 \sqrt{s_{X}-d_{X}}+\sqrt{s_{C}-d_{C}}\right) .
$$

The change in shear traction at a point $\mathrm{X}$ between $\mathrm{C}^{(T)}$ and $\mathrm{B}^{(S)}$ is given by subtracting Eq. (25) from Eq. (26),

$$
\begin{aligned}
\Delta q_{X C} & =f\left(K_{N}^{X} \sqrt{s_{X}}-2 K_{N}^{X} \sqrt{s_{X}-d_{X}}+K_{N}^{X} \sqrt{s_{C}-d_{C}}\right)-\left(-f K_{N}^{C} \sqrt{s_{C}}+f K_{N}^{C} \sqrt{s_{C}-d_{C}}\right) \\
& \simeq f K_{N}^{X}\left(\sqrt{s_{X}}+\sqrt{s_{C}}-2 \sqrt{s_{X}-d_{X}}\right),
\end{aligned}
$$

where $s_{X}=s_{C}+\Delta a$ and the change in the normal stress intensity factor is again neglected, $K_{N}^{X} \simeq K_{N}^{C}$. The terms in Eq. (27) may be again expanded in a binomial series and the higher order terms of $d_{X}$ and $\Delta a$ neglected, similar to the procedure adopted in Eq. (22) (i.e. these quantities are assumed to be much smaller than the average contact size $\left.a\left(P_{0}\right)\right)$. By equating Eq. (1) and Eq. (27), the approximate size of the slip zone when point X reaches B is given by

$$
d_{B}=\frac{\Delta K_{T}}{f K_{N}^{B}}+\frac{\Delta a_{C B}}{2} .
$$

Note that both the change in the shear stress intensity factor and the contact size are positive between points $\mathrm{C}$ and $\mathrm{B}$, i.e. $\Delta K_{T}>0$ and $\Delta a_{C B}>0$. Similarly, the shear traction at $\mathrm{B}^{(S)}$ is given by Eq. (26) but with the subscript $B$ instead of $X$,

$$
q_{B}(x)=K_{Q}\left(P_{Z}\right) \sqrt{s_{B}-d_{B}}+f K_{N}^{B}\left(\sqrt{s_{B}}-2 \sqrt{s_{B}-d_{B}}+\sqrt{s_{C}-d_{C}} .\right)
$$

\subsection{Steady-state - unloading from B to $C\left(Q_{\max }\right.$ to $\left.Q_{\min }\right)$}

At the maximum load during steady-state cyclic loading the size of the slip zone at $\mathrm{B}^{(S)}$ does not erase all the locked in shear traction due to $\mathrm{C}^{(T)}$. Therefore, in a similar manner to the procedure adopted for the full solution in 
Eq. (13), a fourth term is added to the shear traction equation when unloading from $\mathrm{B}^{(S)}$ and $\mathrm{C}^{(S)}$, which is written in terms of asymptotes as

$$
q_{X}=K_{Q}\left(P_{Z}\right) \sqrt{s_{C}-d_{C}}-f K_{N}^{X}\left(\sqrt{s_{X}}-2 \sqrt{s_{X}-d_{X}}+2 \sqrt{s_{B}-d_{B}}-\sqrt{s_{C}-d_{C}}\right) .
$$

The change in shear traction during this load phase is obtained by subtracting the shear traction at $\mathrm{B}^{(S)}$ from Eq. (30), which may be written as

$$
\Delta q_{X B}=-f K_{N}^{X}\left(\sqrt{s_{X}}+\sqrt{s_{B}}-2 \sqrt{s_{X}-d_{X}}\right) .
$$

Again, the change in the normal stress intensity factor $K_{N}$ between $\mathrm{X}$ and $\mathrm{B}^{(S)}$ is neglected. Equation (31) is equivalent to Eq. (22) and it becomes obvious that the shear traction during the unloading phase is also given by Eq. (23). And at the minimum loading point $\mathrm{C}^{(S)}$ during the steady-state regime, the slip zone size is given by

$$
d_{C}=-\frac{\Delta K_{T}}{f K_{N}^{C}}+\frac{\Delta a_{B C}}{2},
$$

where the variation in contact size and the change in shear multiplier between $B$ and $C$ are negative, $\Delta a_{B C}<0$ and $\Delta K_{T}<0$.

In quantifying the damage due to reciprocating sliding in the contact zone such as in fretting fatigue problems, the main interest is in what happens during the steady-state loading regime, just before the maximum and minimum loading points, $\mathrm{B}$ and $\mathrm{C}$, when the slip zones are at their maximum size. These are given in asymptotic form by Eqs. (28) and (32), respectively. Note that, although the magnitude of $\Delta K_{T}$ is identical in the two equations, but with opposite signs, the position of the two edges of the stick zone, $a_{C}-d_{C}$ and $a_{B}-d_{B}$, will not be precisely the same. That is because of the error associated with approximating the normal multipliers $K_{N}^{B}$ and $K_{N}^{C}$. This means that, in contrast to the solution from the finite problem in Section 2, the second and third terms in Eq. (30) will not cancel out exactly. However, at this point the problem is already in the steady-state, and the shear traction at $\mathrm{C}$ could be thought of as that given by Eq. (19) instead. The size of the slip zone at point $C$ in the steady-state will be given by $a_{C}-\left(a_{B}-d_{B}\right)$ following the same arguments given in Section 2.

\section{Application}

Both the formulations presented in this paper are general and may be applied to any incomplete contact problem. All one needs to know is the contact law of the specific problem to be able to derive the pressure distribution and the normal generalised stress intensity factor $K_{N}$. In order to quantify the quality of the asymptotic approximation, we first need to apply the problem to a specific contact problem: in fact, the quality of the asymptotic approximation will depend on the geometry of the contact and the load path followed.

\subsection{Example problem - Hertzian contact}

We look now at a Hertzian contact as an example problem to compare the asymptotic and full formulations. The contact law for a two dimensional Hertzian cylindrical contact is given by

$$
a^{2}=\frac{4 P R}{\pi E^{*}}
$$

where $R$ is the relative radius of curvature and $E^{*}$ the plane strain elastic modulus [21]. The contact pressure for the Hertzian case is given by

$$
p(x, P)=\frac{2 P}{\pi a^{2}} \sqrt{a^{2}-x^{2}} .
$$

From Eq. (4), we obtain immediately that

$$
K_{N}=\sqrt[4]{\frac{P}{\pi}\left(\frac{E^{*}}{R}\right)^{3}} .
$$




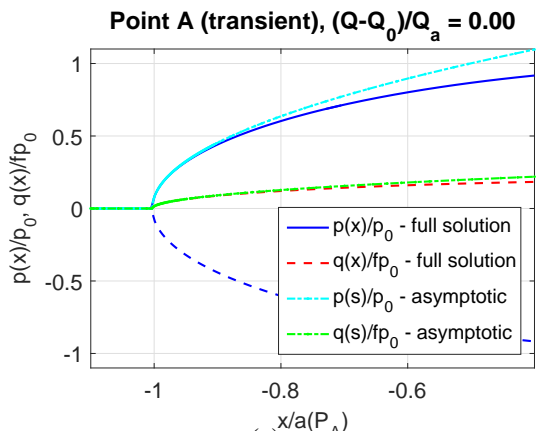

(a) ${ }^{x / a}\left(P_{A}\right)$

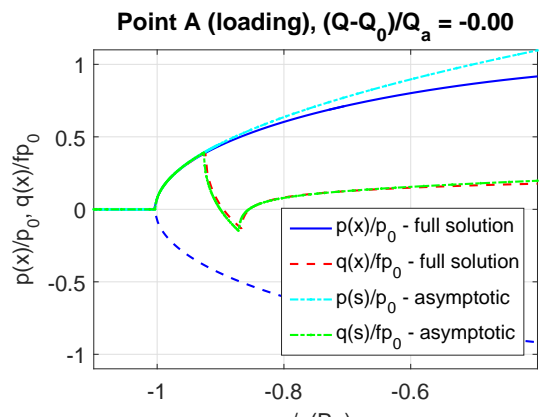

(d) ${ }^{x / a\left(P_{A}\right)}$
Point $B$ (transient), $\left(Q-Q_{0}\right) / Q_{a}=1.00$

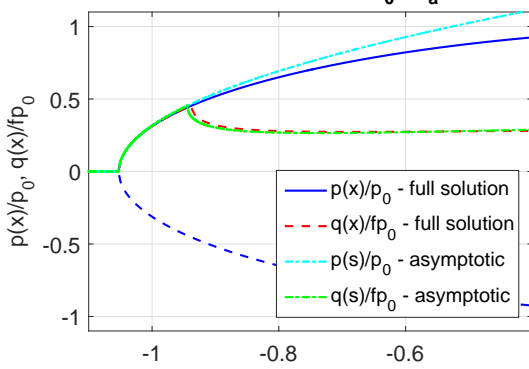

(b) ${ }^{x / a\left(P_{A}\right)}$

Point B (steady-state), $\left(Q-Q_{0}\right) / Q_{a}=1.00$

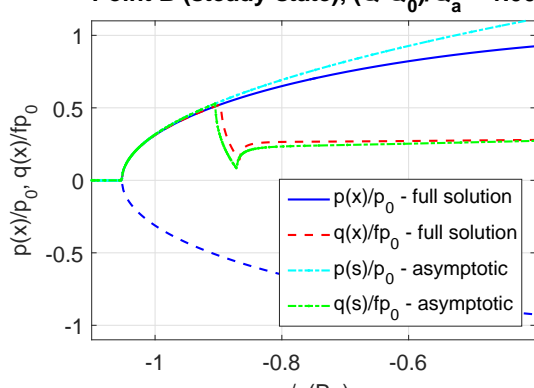

(e) $)^{x / a\left(P_{A}\right)}$

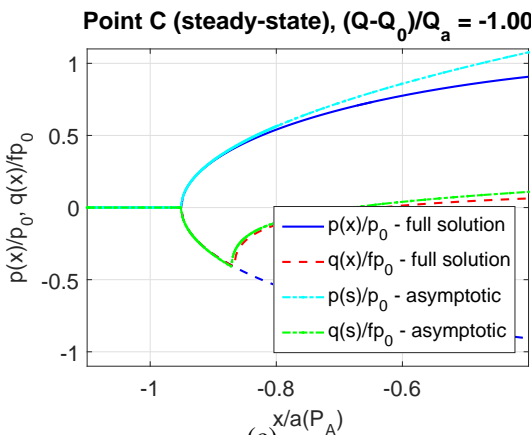

(c) $)^{x / a}\left(P_{A}\right)$

Point A (unloading), $\left(Q-Q_{0}\right) / Q_{a}=0.00$

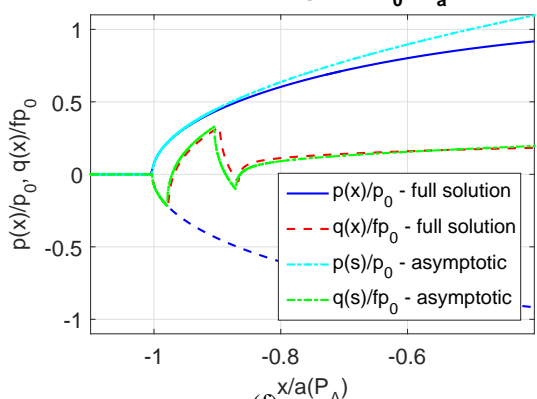

(f) ${ }^{\mathrm{X} / \mathrm{a}\left(\mathrm{P}_{\mathrm{A}}\right)}$

Figure 5: Asymptotic and full contact tractions of an example problem with $P_{a} / P_{0}=0.1, Q_{a} / f P_{0}=0.2, f=0.5$ and $Q_{0} / P_{0}=0.2$ at different points in the loading cycle.

The contact tractions obtained with both the full and asymptotic solutions are presented in Fig. 5. The tractions in the transient cycle at the initial point $\mathrm{A}$ and then at the point of maximum loading B (see Fig. 2) are given in Figs. 5a and $5 \mathrm{~b}$, respectively. Note that at point $\mathrm{A}$ the asymptote representing the shear traction is square root bounded and given by the bounded multiplier $K_{Q}$. In the steady-state cycle, the shear tractions obtained with the full and asymptotic solutions at the point of minimum load $\mathrm{C}$, passing through A during the loading phase, at the maximum load B, and passing though A during the unloading phase, are given in Figs. 5c to 5f, respectively. The quality of the asymptotic formulation will be discussed in Section 5.

\subsection{The steady-state reverse and forward slip zones}

As we are mainly concerned with what happens at the limiting values of loading, it is useful to rewrite the size of the slip zone of the full solution specifically for points B and C, i.e. maximum and minimum loading points, during the steady-state periodic loading in their simplest possible form. The permanent size of the stick zone in the steady-state is given by $a_{K}=a\left(P_{K}\right)$ (Fig. 6). Point $P_{K}$ may also be written as the intersection of a line with slope $f$ passing through $\mathrm{B}$ with a line of slope $-f$ passing through $\mathrm{C}$ (Fig. 4). The point of intersection, $P_{K}$, may be written as

$$
P_{K}=\frac{f\left(P_{B}+P_{C}\right)-\left(Q_{B}-Q_{C}\right)}{2 f}=P_{0}-\frac{Q_{a}}{f}
$$

where $P_{B}=P_{0}+P_{a}, P_{C}=P_{0}-P_{a}, Q_{B}=Q_{0}+Q_{a}$ and $Q_{B}=Q_{0}-Q_{a}$.

The size of the slip zone in the steady-state is $d_{B}=a_{B}-a_{K}$ at the maximum load point $B$, and it can be rewritten as

$$
\frac{d_{B}}{a_{B}}=1-\frac{a_{K}}{a_{B}}
$$

For the specific case of a Hertzian problem, where the size of the contact is given by Eq. (33), Eq. (37) may be written 


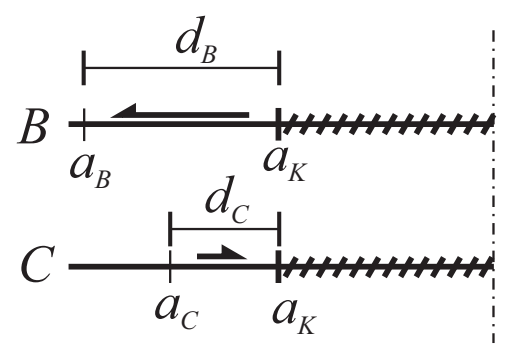

Figure 6: Slip and stick zones at the maximum and minimum loading points during the steady-state loading cycle

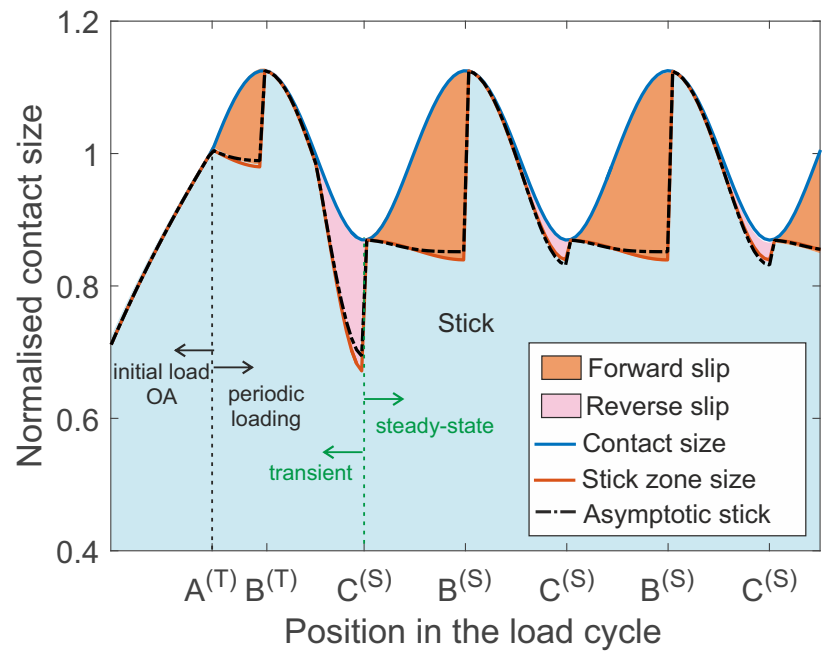

Figure 7: Contact and stick zone sizes asymptotic prediction $\left(P_{a} / P_{0}=0.25, Q_{0}=0, Q_{a} / f P_{0}=0.3\right)$.

as

$$
\frac{d_{B}}{a_{B}}=1-\sqrt{\frac{1-Q_{a} / f P_{0}}{1+P_{a} / P_{0}}} .
$$

Similarly, the normalised slip zone size at the minimum load point $\mathrm{C}, d_{C} / a_{C}$, is given by Eq. (38) but with $1-P_{a} / P_{0}$ in the denominator instead.

The size of the permanent stick zone and the evolution of reverse and forward slip zones at different instants in the load cycle is presented in Fig. 7. Note that there is a zone in the contact that will never slip, another that will slip in the transient phase but not in the steady-state phase, and zones that will experience both forward and reverse slip. In contrast to the problem studied in [22], where the loading was out-of-phase $(\phi \neq 0)$, there is no zone in the contact that experience only forward slip. However, the forward and reverse slip displacements experienced by points in the contact which also experience separation may not be the same.

\section{Quality of the approximation}

The sizes of the slip zone at their maximum extents - just before the turning points in Fig. 2, were calculated using the full expressions and also deduced from the asymptotic representation of the problem. The results found are portrayed in Fig. 8, where the parameter held constant is the ratio of the amplitude of normal load to its mean value $\left(P_{a} / P_{0}\right)$, while the ratio $Q_{a} / f P_{0}$ is varied. The results show the variation in the slip zone size with these two dimensionless quantities for both the maximum (blue lines) and minimum (red lines) load points (i.e. points B and C) in the steady-state loading. The black line is representative of the standard Cattaneo-Mindlin problem when the normal load is held constant, $P_{a} / P_{0}=0$. The solid lines represent the variation of the slip zone size obtained by the 


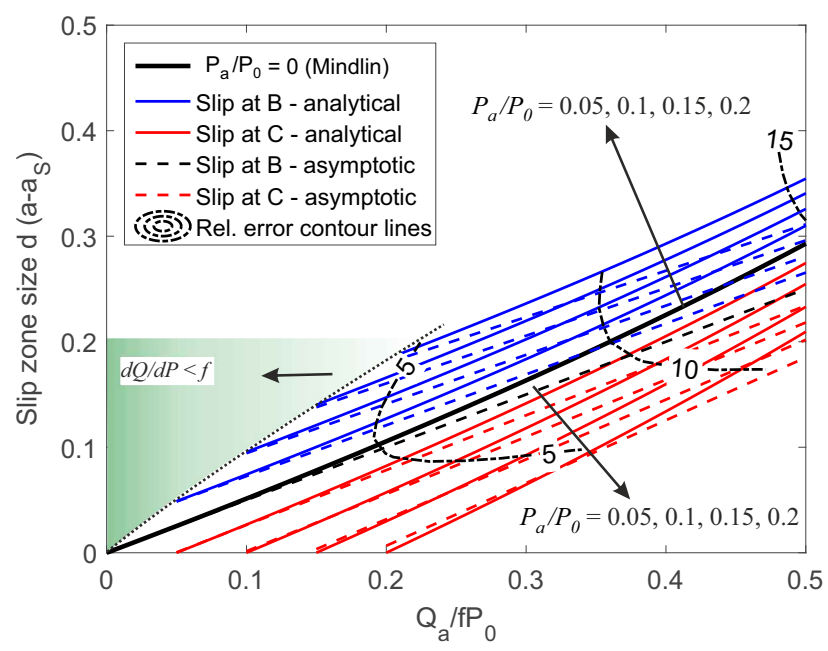

Figure 8: A comparison of the steady-state slip zone sizes at the extremes of loading (point B maximum load, point C, minimum load, see Figs. 2 and 6) for a Hertzian contact between the full solution at that implied by the asymptotic representation, as a function of $P_{a} / P_{0}$.

full solution of the problem given in Section 2, while the dashed lines are the prediction of the asymptotic approach in Section 3. The green region in the bottom left hand side of Fig. 8 represents the region in which the solution is not applicable, since the inclination of the loading path in $P-Q$ space violates the partial slip condition, $\mathrm{d} Q / \mathrm{d} P<f$. Indicative contours of the discrepancy between the two sets of results are also shown in the figure.

Intuitively, we had thought that the quality of the approximate results (from the asymptote) would fall quickly as the ratio $P_{a} / P_{0}$ was increased. This is because the normal asymptote is being asked to reach increasingly far into the interior of the contact, and also the assumption used with the Ciavarella-Jäger theorem that the normal load on the corrective result be constant is increasingly strained. Perhaps surprisingly, this is not what we see. It is clearly the case that, as the shearing force increases in size the difference between the exact and asymptotic solutions increases, and this is to be expected, but it seems that, for a given ratio $Q_{a} / f P_{0}$ the quality of the asymptote actually seems to improve when the contact is changing size, at least for moderate changes of contact size. And, furthermore, this is true at both the maximum and minimum sizes of the contact. At the minimum size of the contact, the error due to the variation in normal load represents an overestimate of the slip zone size, while the increase in $Q_{a} / f P_{0}$ underestimates the slip zone size. Hence, there is an improvement in the asymptotic prediction for moderate values of $Q_{a} / f P_{0}$ before an increase in error for larger values of $Q_{a} / f P_{0}$. At the maximum contact size, the predicted slip zone size underestimation increases with the increase of both $Q_{a} / f P_{0}$ and $P_{a} / P_{0}$. The apparent improvement in the relative error of the asymptotic prediction for lower values of $Q_{a} / f P_{0}$ in Fig. 8 at the maximum contact size point is therefore due to the increase in size of the contact as a whole.

The error implicit in the asymptotic prediction of the slip zone size may be estimated by expanding the exact solution of the permanent size of the slip zone given in Eq. (38) in a binomial series and comparing the linear term with the asymptotic formulation (a similar procedure was done in [16] for the constant normal load problem). This analysis is problem specific and here is done for the Hertzian contact example. The expansion of Eq. (38) in a binomial series is

$$
\frac{d_{B}}{a_{B}} \simeq \frac{1}{2} \frac{Q_{a}}{f P_{0}}+\frac{1}{2} \frac{P_{a}}{P_{0}}-\frac{1}{4} \frac{Q_{a}}{f P_{0}} \frac{P_{a}}{P_{0}}+\frac{1}{8}\left(\frac{Q_{a}}{f P_{0}}\right)^{2}-\frac{3}{8}\left(\frac{P_{a}}{P_{0}}\right)^{2}+\ldots
$$

Furthermore, the asymptotic slip zone size estimation at the maximum load point in the steady-state cyclic loading is given by Eq. (28). Dividing Eq. (28) by $a_{B}$ and substituting the value of $K_{N}, \Delta K_{T}$ and $\Delta_{a}$, the asymptotic slip zone 
size is given by

$$
\begin{aligned}
\frac{d_{B}}{a_{B}} & =\frac{1}{2} \frac{Q_{a}}{f\left(P_{0}+P_{a}\right)}+\frac{1}{2}\left(1-\sqrt{\frac{P_{0}-P_{a}}{P_{0}+P_{a}}}\right) \\
& \simeq \frac{1}{2} \frac{Q_{a}}{f P_{0}}+\frac{1}{2} \frac{P_{a}}{P_{0}}-\frac{1}{2} \frac{Q_{a} P_{a}}{f P_{0}^{2}}-\frac{1}{4}\left(\frac{P_{a}}{P_{0}}\right)^{2}+\ldots
\end{aligned}
$$

where Eq. (40) was also expanded in a binomial series, by taking $P_{a} \ll P_{0}$. Although the quadratic terms of Eqs. (39) and (40) are not identical, the linear terms of both equations are identical and for small value of $P_{a} / P_{0}$ and $Q_{a} / f P_{0}$ both approaches will give similar results. In fact, as shown in Fig. 8, the error remains below $10 \%$ as long as the ratio $Q_{a} / f P_{0}<0.3$ and $P_{a} / P_{0}<0.2$. Note that, if there is no change in the normal load, $P_{a} / P_{0}=0$, Eq. (39) is identical to Eq. (26) in [16].

\section{Asymptotic formulation of the slip displacements}

The relative tangential displacement (slip), $g(x)$, in uncoupled incomplete contacts may be obtained from the singular integral equation [20]

$$
\frac{\partial g(x)}{\partial x}=\frac{2}{\pi E^{*}} \int_{-a}^{a} \frac{q(\xi)}{x-\xi} d \xi .
$$

In order to obtain the gradient of relative displacements from the asymptotic formulation at the left hand edge of contact, first, a change of variable needs to be carried out, i.e. $s=x+a, \zeta=\xi+a$. Next, we take the limit $a \rightarrow \infty$, such that Eq. (41) at the maximum load point B becomes

$$
\begin{aligned}
\frac{\partial g(s)}{\partial s} & =\frac{2}{\pi E^{*}} \lim _{a \rightarrow \infty} \int_{0}^{2 a} \frac{q(\zeta)}{s-\zeta} d \zeta=\frac{2 f K_{N}}{\pi E^{*}} \lim _{a \rightarrow \infty} \int_{0}^{2 a}\left(\frac{\sqrt{\zeta}}{s-\zeta}+\frac{\sqrt{\zeta-d}}{s-\zeta}\right) d \zeta, \\
& =\frac{2 f K_{N}}{E^{*}} \sqrt{d-s} .
\end{aligned}
$$

The slip itself is obtained by integrating Eq. (42) as

$$
\begin{aligned}
\left|g_{\text {Asym }}(t)\right| & =\frac{2 f K_{N}}{E^{*}} \int_{0}^{t} \sqrt{\tau} d \tau, \quad \text { where } t=d-s, \\
& =\frac{2 f K_{N}}{E^{*}} \frac{2}{3}(d-s)^{3 / 2} .
\end{aligned}
$$

A comparison of the solution of the slip given by Eq. (43) for the Hertzian problem, where $K_{N}=1 / 2 p_{0} \sqrt{8 / a}$, and the solution of the full two-dimensional Hertz problem given in [23] is presented in Fig. 9. The load ratio $Q / P$ of the example problem presented is in the limit of the range of application of the asymptotes with a load ratio $Q / P=0.33$. Nevertheless, a good approximate solution of the slip may be obtained with the asymptotic formulation as long as the slip zone is small when compared to the contact size. Equation (43) may be applied to any incomplete contact provided the appropriate $K_{N}$ is used. Once the slip displacements and shear traction in the slip zone are known, one can obtain the energy dissipated per cycle and damping factor for estimating, for example, the fretting damage.

\section{Conclusions}

This paper represents further progress in our ambition to produce a full theory for the partial slip contact problem for any convex solids pressed together and subject to varying normal as well as shear load and, although not explicitly treated here, differential bulk tension in the bodies. The paper represents a simpler loading trajectory than that studied by Barber et al. [10], but it has the advantages of simplicity and that the same two points on the loading trajectory (the ends of the path) represent states where simultaneously the contact is at the extremes of size and the slip zones take their maximum value. It is also possible with this simple loading trajectory to write closed form solutions for 


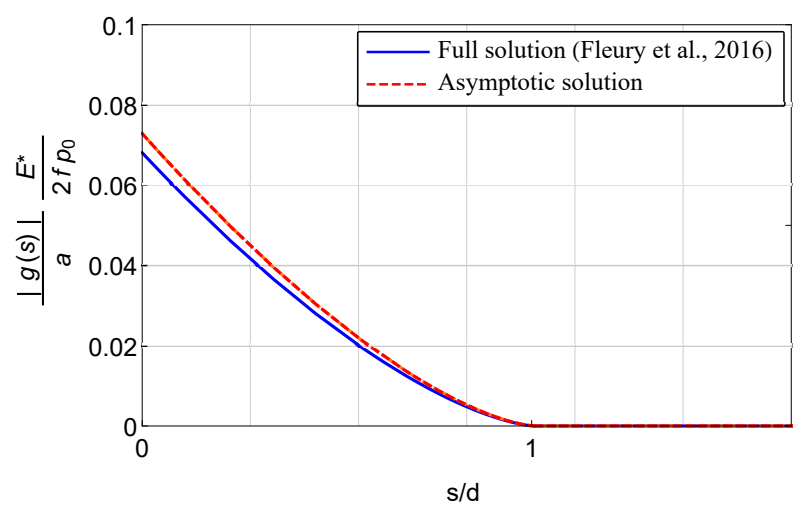

Figure 9: Comparison between the approximate slip distribution obtained by the asymptotic approach and the full solution of a Hertz problem derived in [23] for a load ratio $Q / P=0.33$.

the extent of the steady-state slip zones, and which must be responsible for controlling the extent and magnitude of fretting damage.

We have two reasons for introducing the concepts of local contact edge asymptotes. One is analytical: the procedure adopted in Section 2 and in [10] for determining the traction distributions at various points in the cycle hinges on the slip zones being of the same sign. The procedure simply cannot work if the slip zones are of opposite sign and, in the presence of all sensible histories of bulk tension, at least some points in the loading cycle this is encountered. However, by concentrating our attention on each end separately this objection is removed, and reversing of the slip direction poses no obstacles to using a slightly developed form of the procedure. The second reason for developing the asymptotic representation is that these self similar quantities (particularly that relating to shear) have been shown to be responsible for controlling fretting damage and the nucleation of fretting fatigue cracks.

The asymptotic method aims at providing better tools to increase the characterisation of the tractions at the edge of incomplete contacts, but it does not answer several other questions in fretting fatigue, such as micro-structural and surface finishing effects. These are relevant in the crack initiation process. Nevertheless, the method can significantly improve the correlation of fretting fatigue data when compared with traditional stress based methods [14, 15], although it is imperative to obtain further experimental data for a definite validation of the generalised stress intensity factors as correlation parameters. The representation of the contact tractions near the edge in terms of the two quantities, $K_{N}$ and $K_{T}$, and their respective variations, provides several important characteristics of the contact itself, such as the slip zone size and the amount of slip throughout the load cycle, but also the stresses and the stress concentration under the edge of the contact. Although $\Delta K_{T}$ has been shown to be a good parameter for correlation with fretting fatigue lives when the normal load is kept constant, it cannot alone characterise the stress concentration under the contact [24]. Hence, it is important to include the normal scaling factor, $K_{N}$, as well. Not only is the multiplier $K_{N}$ necessary to determine the size of the slip zone and amount of slip (providing, hence, some quantification of the fretting damage), but it also changes the stresses under the contact, allowing the effect of the normal load to be taken into account.

The 'down side' to the adoption of asymptotic solutions is that they are only approximate, and here we choose to use just the first term in the series. The one describing contact pressure directly, also indirectly, affects our prediction of the slip zone size and is, at some points in the loading cycle, defined from a point located at the maximum extent of contact. However, it is used to infer what happens when the contact shrinks, and therefore the quality of the approximation deteriorates. It follows that the whole procedure works best when the normal load is constant, and becomes increasingly poor when the range of normal load is increased.

\section{References}

[1] C. Cattaneo. Sul contatto di due corpi elastici: distribuzion local degli sfozi. Rend. Accad. Naz. Lincei, 27:342-348,434-436,474-478, 1938.

[2] R. D. Mindlin. Compliance of elasic bodies in contact. J. Appl. Mech. Trans. ASME, 16:249 - 268, 1949.

[3] R. D. Mindlin, W. P. Mason, T. F. Osmer, and H. Deresiewicz. Effects of an oscillating tangential force on the contact surfaces of elastic spheres. Proc. First Nat Congress of Appl Mech, pages 203-208, 1951.

[4] R. D. Mindlin and H. Deresiewicz. Elastic spheres in contact under varying oblique forces. Trans. ASME J. Appl. Mech., 20:372-344, 1953. 
[5] L. A. Galin. Pressure of a punch with friction and cohesion domains. PMM J. Appl. Math. Mech., 9:413-424, 1945.

[6] L. A. Galin and G. M. L. Gladwell. Contact problems: the legacy of L.A. Galin, volume 155. Springer Science \& Business Media, 2008.

[7] D Nowell and D. A. Hills. Mechanics of fretting fatigue tests. Int. J. Mech. Sci., 29(5):355-365, 1987.

[8] J. Jäger. Half-planes without coupling under contact loading. Arch. Appl. Mech., 67:247 - 259, 1997.

[9] M. Ciavarella. The generalized cattaneo partial slip plane contact problem. itheory. Int. J. Solids Struct., 35(18):2349 - 2362, 1998.

[10] J. R. Barber, M. Davies, and D. A. Hills. Frictional elastic contact with periodic loading. Int. J. Solids Struct., 48:2041 - 2047, 2011.

[11] D. Dini and D. A. Hills. Bounded asymptotic solutions for incomplete contacts in partial slip. Int. J. Solids Struct., 41(24):7049-7062, 2004.

[12] D. Dini, A. Sackfield, and D. A. Hills. Comprehensive bounded asymptotic solutions for incomplete contacts in partial slip. J. Mech. Phys. Solids, 53(2):437-454, 2005.

[13] A. Sackfield, A. Mugadu, J. R. Barber, and D. A. Hills. The application of asymptotic solutions to characterising the process zone in almost complete frictionless contacts. J. Mech. Phys. Solids, 51(7):1333-1346, 2003

[14] D. A. Hills and D. Dini. A new method for the quantification of nucleation of fretting fatigue cracks using asymptotic contact solutions. Tribology international, 39(10):1114-1122, 2006.

[15] D. A. Hills, A. Thaitirarot, J. R. Barber, and D. Dini. Correlation of fretting fatigue experimental results using an asymptotic approach. Int. J. Fatigue., 43:62 - 75, 2012.

[16] D. A. Hills, R. M.N. Fleury, and D. Dini. Partial slip in complete contacts under constant normal load and subject to periodic loading. Int. J. Mech. Sci., 108-109(5):115-121, 2016.

[17] R. M. N. Fleury, D. A. Hills, and J. R. Barber. A corrective solution for finding the effects of edge rounding on complete contact between elastically similar bodies. part i: Contact law and normal contact considerations. Int. J. Solids Struct., 85 - 86:89-96, 2016.

[18] R. M. N. Fleury, D. A. Hills, and J. R. Barber. A corrective solution for finding the effects of edge rounding on complete contact between elastically similar bodies. part ii: Near-edge asymptotes and the effect of shear. Int. J. Solids Struct., 85-86:97 - 104, 2016.

[19] D. A. Hills, R. M.N. Fleury, R. Ramesh, and K. Parel. A unified approach for representing fretting and damage at the edges of incomplete and receding contacts. Tribology Int., 2016. In press, http://dx.doi.org/10.1016/j.triboint.2016.08.026.

[20] D. A. Hills and D. Nowell. Mechanics of Fretting Fatigue. Kluwer Academic Publishers, United Kingdom, 1994.

[21] K. L. Johnson. Contact Mechanics. Cambridge University Press, 1985.

[22] M. Davies, J. R. Barber, and D. A. Hills. Energy dissipation in a frictional incomplete contact with varying normal load. Int. J. Mech. Sci., $55(1): 13-21,2012$.

[23] R.M.N. Fleury, R.J.H. Paynter, and D. Nowell. Estimation of the coefficient of friction in partial slip contacts between contacting nickel superalloys. Tribology Int., 2016. In press, http://dx.doi.org/10.1016/j.triboint.2016.09.039.

[24] M Ciavarella and D Dini. A refined clna model in fretting fatigue using asymptotic characterization of the contact stress fields. Fatigue Fract. Eng. M., 28(12):1099-1112, 2005.

\section{Appendix A. Contact law and contact edge direct traction}

The Mossakovski-Barber half-plane formulation for half-plane contact problems hinges on the representation of a convex (symmetrical) contact as a set of flat punches. Starting from the result that the contact pressure distribution

$$
p(x)=f_{0}(x, a)
$$

where

$$
f_{0}(x, a)=\left\{\begin{array}{cc}
\frac{1}{\sqrt{a^{2}-x^{2}}} & -a<x<a \\
0 & |a|
\end{array}\right.
$$

gives rise to surface displacement where the slope is given by

$$
\frac{\mathrm{d} v}{\mathrm{~d} x}=\frac{\kappa+1}{2 \mu} h_{0}(x) .
$$

where $\mu$ is the modulus of rigidity, $\kappa$ is Kolosov's constant and

$$
h_{0}(x)=\left\{\begin{array}{cc}
0 & -a<x<a \\
\frac{-\operatorname{sgn}(x)}{\sqrt{a^{2}-x^{2}}} & |x|>a
\end{array} .\right.
$$

So, by superposition, if we have a set of punches of density $F(s)$, we can see that we can separately integrate the two results just quoted, with careful attention to the limits of integration, to show that a punch giving rise to a pressure distribution $p(x, a)$, where

$$
\left.\left.p(x, a)=\int_{0}^{a} F\right) s\right) f_{0}(x, s) d s
$$


gives rise to a surface slope

$$
\frac{\mathrm{d} v}{\mathrm{~d} x}=\frac{\kappa+1}{2 \mu} \int_{0}^{s} F(s) h_{0}(s) d s
$$

and the corresponding normal load on the punch is given by

$$
P(a)=\pi \int_{0}^{a} F(s) d s
$$

We now have enough of a structure to derive the result we need. First, if we differentiate the last result with respect to $a$ we have that

$$
F(a)=\frac{\mathrm{d} P}{\mathrm{~d} a}
$$

and, from our definition of $K_{N}$ we see that

$$
\frac{\partial p(x, a)}{\partial d}=\frac{\mathrm{d} K_{N}}{\mathrm{~d} a} \sqrt{a-x}+\frac{K_{N}}{2 \sqrt{a-x}},
$$

so that

$$
K_{N}=\lim _{x \rightarrow a^{-}} 2 \sqrt{a-x} \frac{\partial p(x, a)}{\partial a} .
$$

Now, differentiating the expression for the punch contact pressure, we see that

$$
\pi \frac{\partial p(x, a)}{\partial a}=\frac{F(a)}{\sqrt{a^{2}-x^{2}}} .
$$

Therefore

$$
K_{N}=\frac{2 \sqrt{a-x}}{\pi} \frac{F(a)}{\sqrt{a^{2}-x^{2}}}=\frac{F(a)}{\pi} \sqrt{\frac{2}{a}},
$$

and thus

$$
K_{N}=\frac{1}{\pi} \sqrt{\frac{2}{a}} \frac{d P}{d a}
$$

\section{Appendix B. Ciavarella-Jäger theorem applied to asymptotes}

Barber et al. [10] devised a simple notation for expressing the result embodied in the Ciavarella-Jäger formulation. This is that, if the current normal load is $P_{2}$, stick is preserved over a region $A\left(P_{1}\right)$ by a shear traction distribution given by

$$
q(x)=f p\left(x, P_{2}\right)-f p\left(x, P_{1}\right),
$$

where $f$ is the coefficient of friction. The notation implies that terms in the expression are included only when the value of $x$ falls within the contact zone specified. Thus, the second term is included in the equation just given only if $x \in A\left(P_{1}\right)$. We introduce the notation that, adjacent to the edge of the contact, the contact pressure may be written down in the following form

$$
p\left(x, K_{N}^{1}\right)=K_{N}^{1} \sqrt{a_{1}-x} .
$$

It follows that the shear traction distribution at a higher load $K_{N}^{2}$ for a partial slip contact may be written in the form

$$
q(x)=f K_{N}^{2} \sqrt{a_{2}-x}-f K_{N}^{1} \sqrt{a_{1}-x},
$$

where the second term makes no contribution if $a_{1}<x<a_{2}$. At this point the result is general and could be applied to any contact form capable of idealisation by a half-plane. But now let us make it specific to a Hertzian contact, so 
that the contact law and calibration for the generalised stress intensity factors take the form

$$
P=\frac{\pi E^{*} a^{2}}{4 R}
$$

and

$$
K_{N}=\frac{E^{*}}{R} \sqrt{\frac{a}{2}} .
$$

We can therefore write down the shear traction distribution for a Hertzian contact in partial slip as

$$
\frac{q(x)}{f K_{N}^{2}}=\sqrt{a_{2}-x}-\frac{K_{N}^{1}}{K_{N}^{2}} \sqrt{a_{1}-x}=\sqrt{a_{2}-x}-\sqrt{\frac{a_{1}}{a_{2}}} \sqrt{a_{1}-x} .
$$

With a finite contact the usual way of obtaining $a_{1}$ would be to impose overall equilibrium parallel with the surface but this is not possible with a semi-infinite form of the problem because the integrals become unbounded. We approach the problem in a different way by noting that, if the contact is fully adhered and all interface slip prevented, the shear traction distribution is given by

$$
q(x)=\frac{K_{T}}{\sqrt{a_{2}-x}} .
$$

The fully stuck and partial slip forms must be the same at points which are both well away from the origin and also well away from the slip zone, i.e. values of $x$ where $a_{1} \gg a_{1}-x \gg a_{1}-a_{2}$. Let $s=a_{2}-x$, so that $a_{1}-x=s-d$, where $d=a_{2}-a_{1}$. Thus

$$
\begin{gathered}
\frac{q(s)}{f K_{N}^{2}}=\sqrt{s}-\sqrt{1-\frac{d}{a_{2}}} \sqrt{s-d} \quad \text { and } \quad q(s)=\frac{K_{T}}{\sqrt{s}} . \\
\frac{q(s)}{f K_{N}^{2}}=\sqrt{s}\left[1-\left(1-\frac{d}{2 s}+\ldots .\right)\right],
\end{gathered}
$$

giving

$$
\frac{K_{T}}{K_{N}^{2}}=\frac{f d}{2}
$$

